\title{
A Theory of Fluctuations in Stock Prices
}

\author{
Ángel L. Alejandro-Quiñones ${ }^{\dagger}$, Kevin E. Bassler ${ }^{\dagger}$, Michael \\ Field $^{\ddagger}$, Joseph L. McCauley ${ }^{\dagger}$, Matthew Nicol $^{\ddagger}$, Ilya \\ Timofeyev ${ }^{\ddagger}$, Andrew Török ${ }^{\ddagger}$, and Gemunu H. Gunaratne \\ ${ }^{\dagger}$ Department of Physics, University of Houston, Houston, TX 77204 and \\ ${ }^{\ddagger}$ Department of Mathematics, University of Houston, Houston, TX 77204
}

\begin{abstract}
The distribution of price returns for a class of uncorrelated diffusive dynamics is considered. The basic assumptions are (1) that there is a "consensus" value associated with a stock, and (2) that the rate of diffusion depends on the deviation of the stock price from the consensus value. We find an analytical expression for the distribution of returns in terms of the diffusion rate, when the consensus value is assumed to be fixed in time. The analytical solution is shown to match computed histograms in two simple cases. Differences that result when the consensus value is allowed to change with time are presented qualitative explanations.
\end{abstract}

\footnotetext{
*Also at The Institute of Fundamental Studies, Kandy 20000, Sri Lanka; Electronic address: gemunu@uh.edu
} 


\section{INTRODUCTION}

Statistical analysis of a wide range of physical and other processes have shown the presence of non-Gaussian distributions. They include temperature fluctuations in hard turbulence [1], diffusion in inhomogeneous media [2, 3], and price variations in financial markets [4, 5]. One common characteristic in these distributions is the presence of exponential or power-law tails, signifying a more frequent occurrence of large deviations than expected from a collection of independent, identically distributed events [6]. The width of the distributions have also been shown to scale as the time interval during which the fluctuations occur. Based on these properties, it has been proposed that Levy distributions be used to describe fluctuations in the underlying processes [7, 8]. In this paper, we propose an alternative explanation for the non-Gaussian distributions, namely a non-uniform diffusion rate [9, 10]. The discussion here is based on fluctuations in financial markets.

Consider a stock whose price at time $t$ is given by $S(t)$. Most financial market analyses are conducted in terms of the "return" of stock, $x(t)=\ln \left[S(t) / S_{0}\right]$, where $S_{0}$ is a reference price. Studies have shown that successive fluctuations in return are uncorrelated [4, 11]. Most theoretical analysis assume further that successive events are statistically independent [6]. The traditional theoretical model uses a simple discrete random walk in the logarithm of price to describe the dynamics of an asset in a market. This model results in a normal, or Gaussian distribution of price returns [12]. On the other hand, empirical data clearly show that the price return distribution of real markets deviates significantly from a Gaussian, especially far from the mean [13]. (For recent reviews see [4, 5, 14, 15, 16, 17, 18].) In particular, some detailed studies 4, 14, 15] have found that the tails of the distribution have an asymptotic power law decay, typically with exponents larger than 2, while others [18, 19] have found that the tails of the distribution are better described by an exponential decay.

It has been conjectured that the non-normality observed in real financial markets can be explained by assuming that although successive events may be uncorrelated, the rate of trading depends on the price of the stock [19]. In particular, it is assumed that there is a "consensus" value $\bar{x}(t)$ of the return, at which point the stochastic fluctuations are minimum, and the level of the fluctuations increase as the return moves away from $\bar{x}(t)$. In other words, the rate of transactions depend on the return. In particular, a large change in price is likely to be followed by large fluctuations. 
Here, we explore the implications of the conjecture by studying variations of the traditional model. Like the traditional model, we use an uncorrelated random walk to represent the dynamics of price returns. However, unlike the former, where the rate of trading is independent of price, our model assumes a rate which increases the deviation of the stock price from $\bar{S}(t)$. It will be demonstrated that this modification in the dynamics can reproduce the range of non-Gaussian behavior of the price return distributions observed empirically in real markets.

If an uncorrelated random walk models fluctuations of price returns, then the continuous dynamics is a diffusive process. In models considered here, the diffusion coefficient $D(x, t)$ is assumed to depend on the time elapsed $t$ and the return $x(t)$. It is assumed further that the dependence on $x(t)$ is a function of its deviation from the consensus return $\bar{x}(t)=\ln \left[\bar{S}(t) / S_{0}\right]$.

The remainder of the paper is structured as follows. First, exact analytical results are presented for the distribution of price returns, valid for an arbitrary functional form of the diffusion coefficient, when $\bar{S}$ is fixed in time. The analytical solutions for the distributions are obtained by solving the Fokker-Planck equation for the corresponding diffusive process. Then, two specific functional forms for $D(x, t)$ are considered. The first form is a piecewise linear function of $u=x / \sqrt{t}$. While the second is a quadratic function of $u$. The form of the distributions are obtained analytically. Results from simulations of the corresponding discrete random walks are also presented and shown to be consistent with the analytical forms. Subsequently, we provide results from computations for a model with time dependent consensus value. In conclusion, we summarize the results and discuss their importance for understanding the behavior of real markets.

\section{EXACT ANALYTICAL SOLUTION FOR THE RETURN DISTRIBUTION}

To obtain an analytical expression for the price return distribution $W(x, t)$ of the diffusive processes we are considering, note that it satisfies the Fokker-Planck equation [21, 22]

$$
\frac{\partial W}{\partial t}=-R(t) \frac{\partial W}{\partial x}+\frac{1}{2} \frac{\partial^{2}}{\partial x^{2}}(D W)
$$

where $D \equiv D(x, t)$ is the diffusion coefficient [23], and $R(t)$ is a (time-dependent) drift

rate [24]. For simplicity, we assume $R(t)=0$ for the rest of this analysis. However, the case of non-zero $R(t)$ can also be treated using a simple coordinate transformation $x^{\prime}=$ 
$x-\int_{0}^{t} R\left(t^{\prime}\right) d t^{\prime}$.

A normalizable solution to Eq. (11), consistent with empirical investigations of financial markets [4, 5, 25], can be found by assuming that the distribution of returns has the scaling form

$$
W(x, t)=\frac{1}{t^{\eta}} F(u) .
$$

Here $u=x / t^{\eta}$, and $\eta$ is the self-similarity exponent [5]. We also assume that the diffusion rate is a function of $u$. This scaling hypothesis leads a unique value for $\eta$, which can be seen by noting that, using it, Eq. (11) becomes

$$
-\frac{\eta}{t^{\eta+1}} F(u)-\frac{\eta}{t^{\eta+1}} u F^{\prime}(u)=\frac{1}{2} \frac{1}{t^{3 \eta}}(D F)^{\prime \prime}(u) .
$$

Consequently $\eta=1 / 2$, a value which consistent with conclusions from empirical studies of real markets. Then Eq. (3) simplifies to

$$
[D(u) F(u)]^{\prime \prime}+[u F(u)]^{\prime}=0
$$

which can be integrated to

$$
[D(u) F(u)]^{\prime}+u F(u)=\text { Const }
$$

If $D(u)$ is symmetric about $u=0$ and the diffusion process starts at the origin, then $F(u)$ will also be symmetric about $u=0$. Under these conditions, both terms in the LHS of Eq. (5) are anti-symmetric about $u=0$, and thus Const $=0$. Therefore,

$$
D(u) F(u)^{\prime}=-\left[u+D(u)^{\prime}\right] F(u)
$$

which has a general solution of the form

$$
F(u)=\frac{1}{D(u)} \exp \left[-\int^{u} \frac{\bar{u}}{D(\bar{u})} d \bar{u}\right] .
$$

As an example, consider a constant diffusion coefficient $D(x, t)=D_{0}$. In this case, Eq. (17) gives a solution of the form

$$
F(u)=C_{0} \exp \left[-\frac{1}{2 D_{0}} u^{2}\right]
$$

which is the well-known result for the traditional model of distribution of returns. 


\section{STATIC CONSENSUS PRICE $\bar{S}$}

In this section we present two models that use a non-constant diffusion coefficient $D(u)$ and take the consensus value $\bar{S}(t)$ to be fixed, without loss of generality, at $\bar{S}(t)=S_{0}$. For both cases we present numerical and analytical results.

\section{A. Piecewise Diffusion}

The first form of $D(u)$ we consider is a piecewise linear function

$$
D(u)=D_{0}(1+\epsilon|u|),
$$

where $D_{0}$ and $\epsilon$ are constant parameters. It should be noted that $D_{0}$ can be eliminated by a suitable rescaling of time. The exact solution to Eq. (11), obtained using Eq. (17), is

$$
F(u)=C_{0} \exp \left[-\frac{|u|}{D_{0} \epsilon}\right](\epsilon|u|+1)^{\alpha-1},
$$

where $\alpha=1 /\left(D_{0} \epsilon^{2}\right)$, the constant $C_{0}$ which normalizes $W(x, t)$ is given by

$$
C_{0}=\frac{\left[1 /\left(D_{0} \epsilon e\right)\right]^{\alpha}}{2 \sqrt{t} \Gamma[\alpha, \alpha]},
$$

and

$$
\Gamma[a, z]=\int_{z}^{\infty} p^{a-1} e^{-p} d p
$$

is the incomplete Gamma function.

In the limit that $\epsilon$ vanishes, $W(x, t)$ becomes a Gaussian. This can be seen from

$$
\ln F(u) \sim\left(\frac{1}{D_{0} \epsilon^{2}}-1\right) \ln (1+\epsilon|u|)-\frac{|u|}{D_{0} \epsilon} \sim \frac{-u^{2}}{2 D_{0}}+O(\epsilon)
$$

and hence

$$
\lim _{\epsilon \rightarrow 0} F(u) \sim \exp \left[-u^{2} / 2 D_{0}\right]
$$

This is because, in that limit, the diffusion coefficient (9) is a constant. As $\epsilon$ increases the tails of the distribution decay slower. We refer the reader to Ref. [19] for a more detailed study of the special case $\epsilon=1 / \sqrt{D_{0}}$ when $F(u)$ is an exponential distribution.

We simulated the price returns using random walks with steps of unit size occurring at non-constant time intervals. The time between steps is $1 / D(x, t)$ [21], where $D(x, t)$ was calculated at every time step. The simulations consisted of many independent walkers, each 
of which started at the origin, and randomly chose the direction of each event to be either an increase or a decrease with equal probability. The walks continued until a maximum time was reached. Fig. 1 compares the analytical and simulation results, showing good agreement between them.

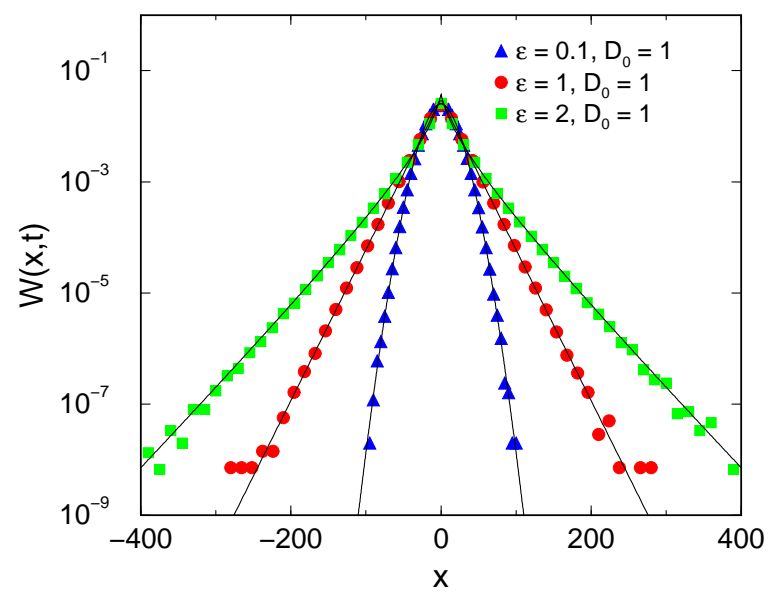

FIG. 1: Distribution of returns for a piecewise linear diffusion coefficient, Eq. (9), with $D_{0}=1$. The shape of the distribution changes with the parameter $\epsilon$. Notice the special cases of $\epsilon=0$ where the distribution is Gaussian, and of $\epsilon=1$ where the distribution is exponential. The solid lines represent the analytical solution of Eq. (10), and the data points are the results of the random walk simulations with $10^{6}$ independent walks, each one lasting a time $t=256$.

\section{B. Quadratic Diffusion}

The second form of $D(u)$ we consider is,

$$
D(u)=D_{0}\left(1+\epsilon u^{2}\right)
$$

It this case, the solution to Eq. (11) obtained using Eq. (77), is

$$
F(u)=\frac{C_{0}}{\left(1+\epsilon u^{2}\right)^{1+\beta}}
$$

where $\beta=1 /\left(2 D_{0} \epsilon\right)$ and the normalization constant for $W(x, t)$ is

$$
C_{0}=\frac{\Gamma[1+\beta]}{\sqrt{t / \epsilon} \Gamma\left[\frac{1}{2}\right] \Gamma\left[\frac{1}{2}+\beta\right]} .
$$


This result is plotted in Fig. 2, where it is compared to the results of the corresponding discrete random walk simulation for different values of $\epsilon$, with $D_{0}=1$. The simulations were performed as in the case of piecewise linear diffusion, except that in this case $D$ is given by Eq. (15). Note also that the results from the simulation are again consistent with the analytical solutions.

As before, the return distribution also becomes a Gaussian in this case when $\epsilon$ vanishes. However, as $\epsilon$ increases, the tails of $W(x, t)$ become power-law distributed. This behavior can better be appreciated in Fig. 3 where a log-log plot for different values of $\epsilon$ is presented. In the limit of $\epsilon \rightarrow \infty$ the tails of the distribution are well fitted by a power-law with exponent 2. Meanwhile, as $\epsilon \rightarrow 0$ the tail can also be fitted with a power-law, but with an exponent whose value increases and $\epsilon$ decreases. However, the fit is good over a range that shrinks as $\epsilon$ decreases. This is expected since the distribution becomes Gaussian in the limit $\epsilon \rightarrow 0$. It is important to point out that as $\epsilon$ is decreased from $\infty$ to 0 the exponent observed in the tail varies from 2 to $\infty$. Thus, these results reproduce the empirical observations of real markets that find fat tailed price return distributions with exponents ranging from 2 upward. Exponents as large as 7.5 have been reported, [4], but at these values the results have large error bars. This is because large exponents are found when the time scale is increased, and the amount of data samples used in the analysis decreases.

Fat tails $f(x, t) \sim|x|^{-\alpha-1}$ for $|x|>>1$, in the range $2<\alpha<\infty$ are also generated by symmetric Lévy distributions [26],

$$
f(x, t)=\frac{1}{2 \pi} \int_{-\infty}^{\infty} d k e^{i k x-|k|^{\alpha} \Delta t}
$$

when $\left\langle\Delta x^{2}\right\rangle=\Delta t^{2 / \alpha}$ for $\alpha>2$, but this implies fractional Brownian motion with Hurst

exponent $H<\frac{1}{2}$. Here, there is no Langevin equation with in $x(D(x, t)$ doesn't exist), and the efficient market hypothesis is violated [27].

\section{DYNAMIC CONSENSUS PRICE $\bar{S}$}

As has already been mentioned, the price of the stock will fluctuate about the consensus value $\bar{S}$. However, contrary to what has thus far been considered, there is no reason to expect that the consensus value should stay fixed. In fact, it is plausible that with every trade $\bar{S}(t)$ will shift by a small amount toward the value of the current price $S(t)$, or equivalently, that 


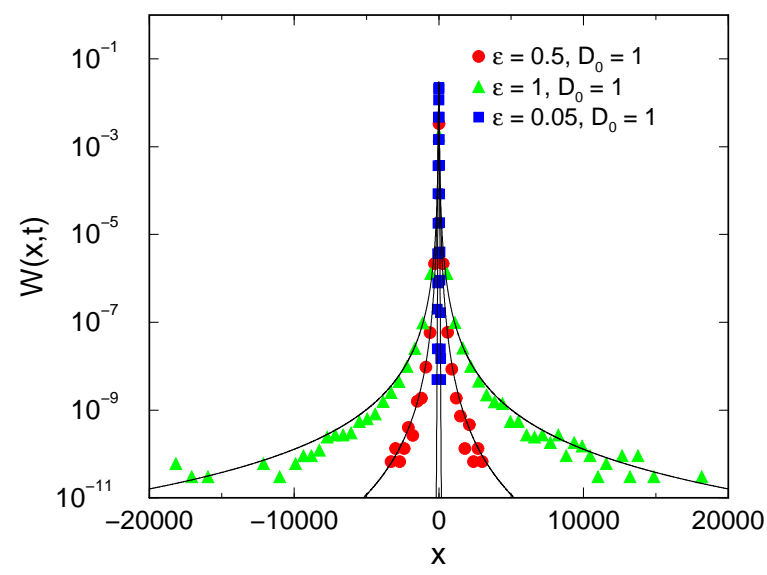

FIG. 2: Distribution of returns using the quadratic diffusion coefficient in Eq. (15). Note that the distribution is not exponential, but instead has fat tails. The solid lines represent the exact solution to Eq. (16), and the data points represent the results from the simulation. For $\epsilon=0.05$ $2 \times 10^{7}$ walks were simulated, $5 \times 10^{7}$ for $\epsilon=0.5$, and $6 \times 10^{7}$ for $\epsilon=1$. The final time used in each case was $t=256$.

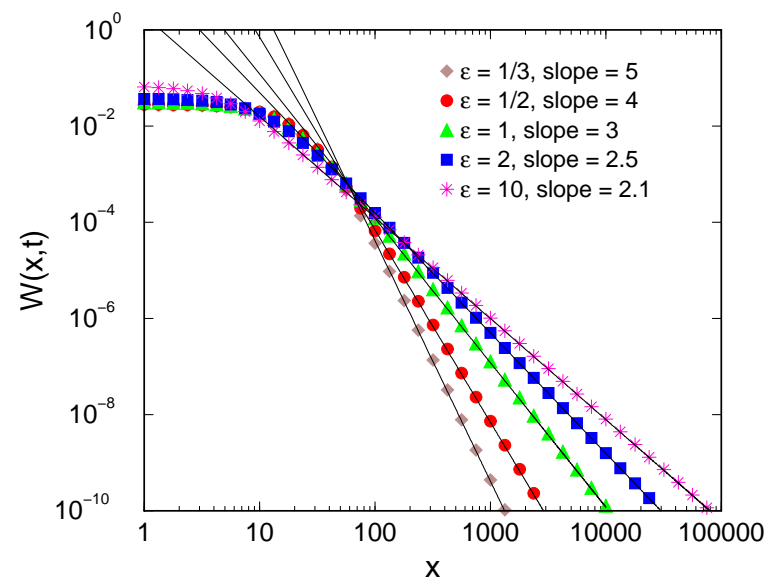

FIG. 3: Log-log plot of the distribution of $x$ using a quadratic $D(x, t)$ showing that the powerlaw tails of $W(x, t)$ can have exponents ranging from 2 upward. The data points represent the analytical solution, from Eq. 16. The tail of each case is fitted with a straight line having a slope equal to the tail exponent.

the consensus value of returns $\bar{x}(t)$ will shift toward the value of the current return $x(t)$. Of course, the diffusion constant will change with the consensus value changes to $D(x-\bar{x}, t)$ in order to keep its minimum at $x=\bar{x}$. 
In this section, results of random walk simulations which utilize the non-constant diffusion coefficients considered in the previous section, and which allow for the consensus value to change, are presented. A very simple dynamics for the value the consensus return $\bar{x}$ is considered; the change in the value at each time step, $\Delta \bar{x}$, is assumed to be proportional to the difference in the current return value and its current value,

$$
\Delta \bar{x}(t)=k[x(t)-\bar{x}(t)]
$$

where $k>0$ is a constant that we will assume to be small. There are two essential differences between the simulations discussed in section III and those in this section. First, as mentioned above the diffusion constant used is $D(x-\bar{x}, t)$ instead of $D(x, t)$. Therefore, the time between steps becomes $1 / D(x-\bar{x}, t)$. Second, the value of $\bar{x}$ is varied dynamically using Eq.(19).

The simulations again begin with the consensus price of the stock equal to its initial value $\bar{S}(0)=S_{0}$, and therefore the initial value of the consensus return vanishes $\bar{x}(0)=0$. Subsequently, $\bar{x}$ will fluctuate around its initial value. Of course, $x(t)$ will also fluctuate about the origin. When the value of $x(t)$ is near the origin, it is often the case that $|x(t)| \approx$ $|\bar{x}(t)|$. This causes the peak in the distribution of returns $W(x, t)$ to smear out.

\section{A. Linear Diffusion}

To understand the effects of a dynamic consensus value $\bar{x}$ on the distribution of price returns, first consider the case of piecewise linear diffusion $D(x-\bar{x}, t)$. As discussed earlier, if $\epsilon=\frac{1}{\sqrt{D_{0}}}$ and $\bar{x}$ is static, this form of the diffusion coefficient will result in an exponential return distribution. Figs. 4 and 6 present the results of simulations with dynamic $\bar{x}$. As expected from the argument in the previous paragraph, the effect of the dynamics of $\bar{x}$ is to smooth out the peak in $W(x, t)$. In fact, it becomes Gaussian in the center, as can be seen from the fit to the quadratic function shown in red in Fig. 4. That function is fit through the 31 points at the peak of $W(x, t)$. The range of the quadratic region is directly related to the value of $k$. If $k$ increases this region is extended to a larger range, see Fig. 5 . Away from the center of the distribution, where the effects of the dynamics of $\bar{x}$ become less important, the exponential form of $W(x, t)$ is retained as expected. This is shown by the fit to the line shown in blue, which works in the tail of the distribution. 


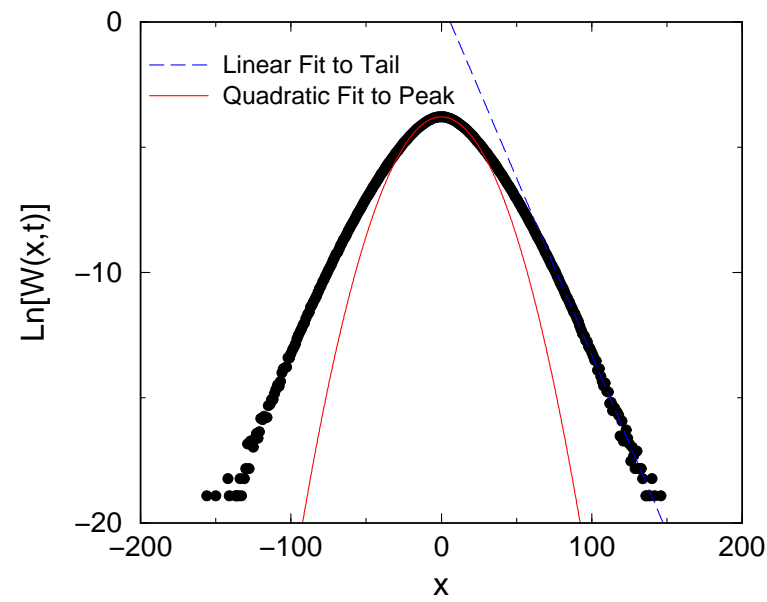

FIG. 4: Distribution of returns with linear diffusion and dynamic consensus value. The center of the distribution of $x(t)$ is well described by a quadratic function. The curve shown was fit to the 31 points in the center. The tails of the distribution have an exponential decay. A straight line was used to fit the tails. The figure shows results for $\epsilon=1, D_{0}=1$ and $k=0.01 .1 .6 \times 10^{8}$ walks, each with a final time of $t=256$, were used in the simulation.

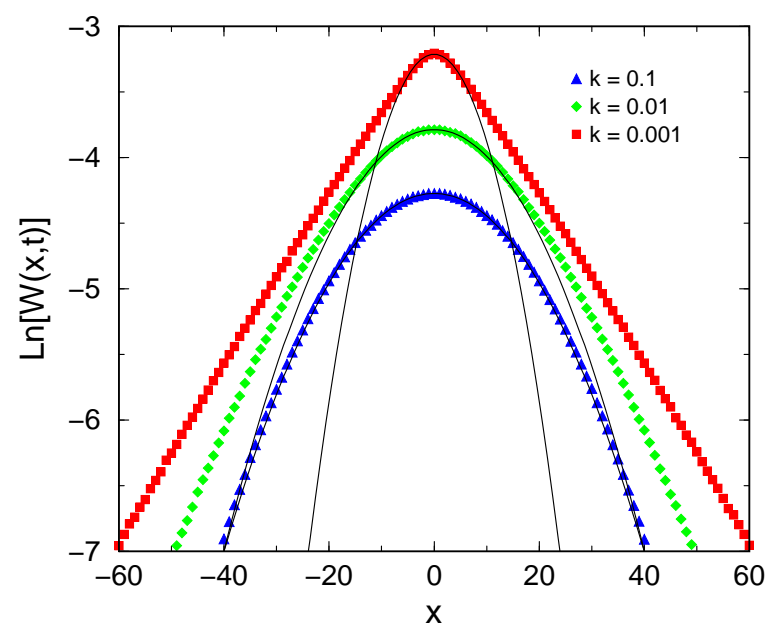

FIG. 5: Dependence on $k$ of the size of the Gaussian region at the center of the distribution. As $k$ increases the width of the Gaussian region is increased. In the limit of $k=1$ the distribution becomes completely Gaussian. The data points represent the simulation and the solid lines represent the quadratic fits. Each simulation consisted of $10^{7}$ random walks, with $\epsilon=1, D_{0}=1$ and $t=256$. For clarity the distributions were shifted vertically and the tails are excluded from the plot. 
Fig. 6] shows the distribution of $\bar{x}$ for different values of $k$, which we will call $P(\bar{x}, t)$ to distinguish it from $W(x, t)$. As $k$ is decreased, $x_{0}$ stays closer to the origin, its starting position, and the tails of the distribution decay rapidly. This is why the tails of the distribution of $x$ are not affected by the movement $\bar{x}$. In the limit of $k=0, \bar{x}$ becomes static, and the distribution will be a single point at the origin. On the other hand, in the limit $k$ goes to 1 the distribution becomes a Gaussian.

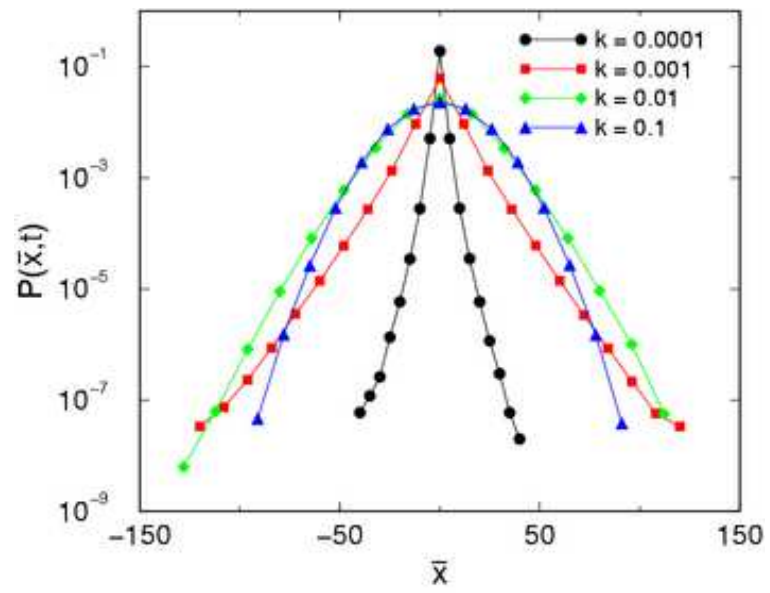

FIG. 6: Distribution of $\bar{x}$ using the piecewise linear diffusion coefficient. Note that the distribution depends on the parameter $k$. For small $k, \bar{x}$ becomes more localized around the origin. In this simulations, $\epsilon=1, D_{0}=1$ and $t=256$ with $10^{7}$ walks each. The solid lines are guides to the eye.

\section{B. Quadratic Diffusion}

Now consider the effects of the dynamics of $\bar{x}$ on the return distribution for the case where $D(x-\bar{x}, t)$ is a quadratic function. In this case, as we have seen, if $\bar{x}$ is static, then the center of the re turn distribution has a peak, but does not have discontinuity in the slope at $x=0$. Fig. $[7$ shows the return distribution calculated from simulations with dynamic $\bar{x}$. As expected, the peak at $x=0$ is broader than in the case of static $\bar{x}$, and it can also be fitted with a quadratic function, indicating a Gaussian peak. Notice, though, the tail behavior in this case differs from that observed when $\bar{x}$ was static. In this case, the tails of the distribution are exponential. We will return to this point at the end of this section.

Fig. 8 shows the distribution of $\bar{x}$. As the value of $k$ decreases, the distribution of $\bar{x}$ becomes sharply peaked. This occurs at the same time that the tails of the distribution are 


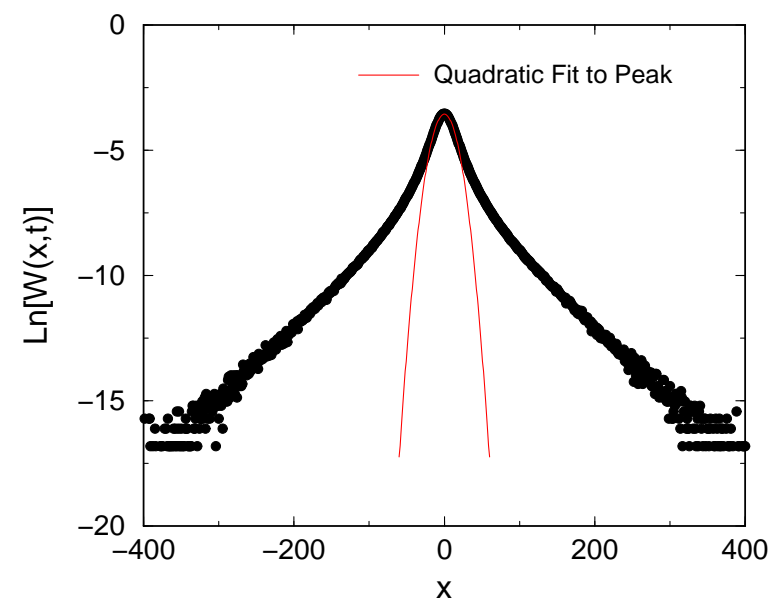

FIG. 7: Distribution of $x$ using a dynamic consensus value and the quadratic diffusion coefficient. The central region of can be fitted using a quadratic function (solid line). To obtain the fitted line, 31 points at the center were used to fit the distribution. The data points show the results from the simulation using $\epsilon=1, D_{0}=1, t=256, k=0.0005$ and $2 \times 10^{7}$ walks.

getting heavier, implying that compared to Fig. [6] there is a larger chance of $\bar{x}$ being far from its original position. This behavior is presumably due to the effect of the fat tails in the distribution of $x(t)$ for static $\bar{x}$. When the value of $x(t)$ is in the tail of its distribution, $\bar{x}$ is "pulled" far away from the origin. This dynamics is very different than what we observed in the case of linear diffusion, where the tails of the distribution of $\bar{x}$ decayed faster as $k$ decreased. Notice that in the limit of $k=0$ the distribution of $\bar{x}$ will also be a single point at the origin, as is also the case for linear diffusion.

We now take a closer look at the tails of $W(x, t)$. Fig. 9] presents a log-log plot with results from simulations for quadratic diffusion using different values of $k$ and $\epsilon=1$. It is observed that as $k$ decreases, the power-law behavior starts to emerge in the tails. In the limit of $k=0$ the results should be the same as in the static $\bar{x}$ case (a power-law with slope 3). To explain why the power-law disappear with an increase of the parameter $k$ we turn to the dynamics of $\bar{x}(t)$. When $k$ is increased the value of $\bar{x}$ will follow closer $x(t)$ making $D(x-\bar{x}, t)$ have a more constant value. This results in the tails of $x(t)$ becoming Gaussian. 


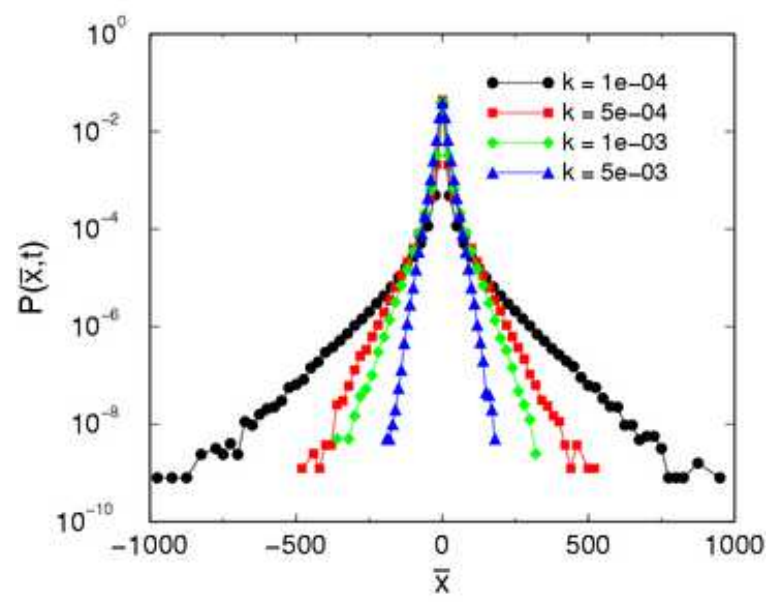

FIG. 8: Distribution of $\bar{x}$ using the quadratic diffusion coefficient with dynamic consensus value. The tails of the distribution get heavier as $k$ is decreased. This results were obtained using $\epsilon=1$, $D_{0}=1$ and $t=256$. The number of walks used was $2 \times 10^{7}$ for $k=5 \times 10^{-3}$ and $k=10^{-3}, 4 \times 10^{7}$ for $k=5 \times 10^{-4}$ and $5 \times 10^{7}$ for $k=10^{-4}$. Here again the solid lines serve as guides to the eye.

\section{CONCLUSIONS}

We have presented a theory for the distribution of stock returns. It is based on the conjecture that the rate of trading of a stock depends on how far its current price is from a consensus price, $\bar{S}$. The resulting models use a non-constant diffusion coefficient $D(x, t)$ to simulate the rate of returns. When $\bar{S}$ is fixed and a piecewise linear coefficient is used, an exponential distribution of returns is found. With quadratic diffusion distributions with fat tails are found. The exponents describing the power law fat tail distributions range from 2 to $\infty$. In both cases we obtained an exact solution for $W(x, t)$ and simulations that support our findings. When $\bar{S}$ is allowed to move, both forms of diffusion coefficient give distributions with an approximately Gaussian near the origin. Finally, we note that the range of behaviors observed here with this simple model covers the range of non-Gaussian behaviors seen in the distribution of returns of real financial markets.

A.L.A. and G.H.G are partially supported by the Institute for Space Science Operations (ISSO) at the University of Houston. K.E.B. is supported by the NSF through grants DMR0406323 and DMR-0427538, and by the Alfred P. Sloan Foundation. G.H.G. is supported 


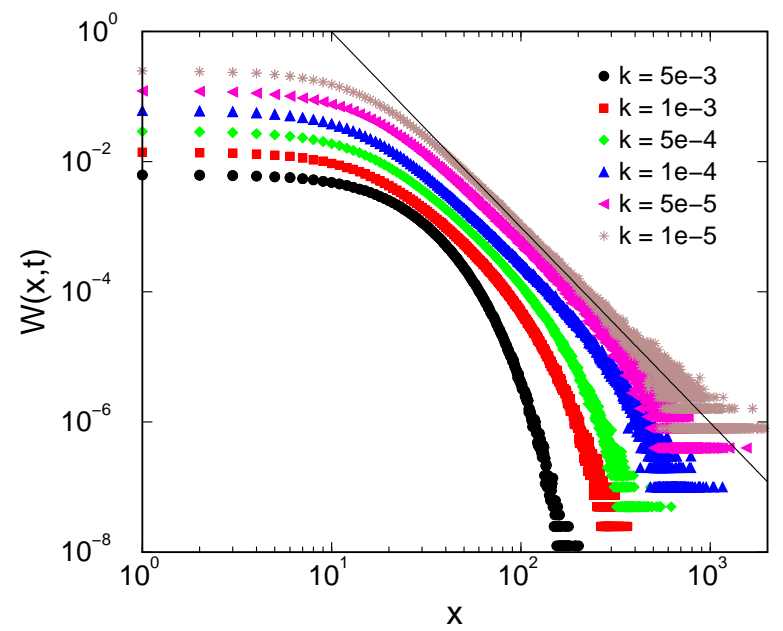

FIG. 9: Log-log plot of the distribution of $x$ with quadratic diffusion coefficient and dynamic consensus value. The fat tails in the distribution go to a power law as $k$ goes to zero. Each simulation used $\epsilon=1, D_{0}=1, t=256$ and $2 \times 10^{7}$ random walks. The black line at the top has a slope $=3$. The distributions have been shifted vertically for clarity.

by the NSF through grant PHY-0202001.

[1] B. Castaing, G. Gunaratne, F. Heslot, L. Kadanoff, A. Libchaber, S. Thomae, X.-Z. Wu, S. Zaleski, and G. Zanetti, J. of Fluid Mech., 204, 1 (1989).

[2] S. Havlin and D. Avraham, Adv. Phy., 36, 695 (1987).

[3] J. W. Hans and K. W. Kehr, Phys. Rep., 150, 263 (1981).

[4] M. M. Dacorogna, R. Gencay, U. Müller, R. B. Olsen, and O. V. Pictet, An Introduction to High-Frequency Finance (Academic Press, San Diego, 2001).

[5] R. N. Mantegna and H. E. Stanley, Nature, 376, 46 (1995); Nature, 383, 587 (1996).

[6] W. Feller, An Introduction to Probability Theory (Wiley, New York, 1957).

[7] J. P. Bouchaud and A. Georges, Physics Report, 195, 127 (1990).

[8] J. Klafter, M. F. Shlesinger, G. Zumofen, Physics Today, Feb., 33 (1996).

[9] N. G. Van Kampen, Stochastic Processes in Physics and Chemistry (North-Holland, Amsterdam, 1981).

[10] R. Friedrich, J. Peinke, and Ch. Renner, Phys. Rev. Lett., 84, 5224 (2000). 
[11] A. Arneodo, J. P. Bouchaud, R. Cont, J. F. Muzy, M. Potters, and D. Sornette, cond-mat/9607120 at lanl.arXiv.org.

[12] M. F. M. Osborne, in: P. Cooter (Ed.), The Random Character of Stock Market Prices (MIT Press, Cambridge, MA, 1964).

[13] B. Mandlebrot, Journal of Business, 36, 392 (1963).

[14] P. Gopikrishnan, V. Plerou, L.A. Nunes Amaral, M. Meyer, H.E. Stanley, Phys. Rev. E, 60, 5305 (1999).

[15] V. Plerou, P. Gopikrishnan, L.A. Nunes Amaral, M. Meyer, H.E. Stanley, Phys. Rev. E, 60, 6519 (1999).

[16] K. Lee, J. Lee, cond-mat/0407418 at lanl.arXiv.org.

[17] H. F. Coronel-Brizio, C. R. de la Cruz-Laso, A. R. Hernandez-Montoya, cond-mat/0303568 lanl.arXiv.org.

[18] J. P. Bouchaud and M. Potters, Theory of Financial Risks (Cambridge University Press, Cambridge, 2000).

[19] J. L. McCauley and G. H. Gunaratne, Physica A, 329, 178 (2003).

[20] J. L. McCauley, Dynamics of Markets: Econophysics and Finance (Cambridge University Press, Cambridge, 2004).

[21] S. Chandrasekar, Rev. Mod. Phys., 15, 1 (1943).

[22] A. D. Fokker, Ann. d. Physik, 43, 812 (1914); M. Planck, Sitz. der preuss. Akad., p. 324 (1917).

[23] Possibilities that include anomalous diffusion have been considered in, for example, Refs. 5] and [25].

[24] Fokker-Planck equations with variable $R$ and $D$ have been used previously to describe dynamics of financial markets, see for example S. Maslov and Y.-C. Zhang, Physica A, 262, 232 (1999) and L. Ramusson and E. Aurell, cs.NI.0102011 at arXiv. Non-linear Fokker-Planck equations have also been proposed, see Ref. [32].

[25] R. Cont, M. Potters, and J. P. Bouchaud, "Scaling in stock market data: stable laws and beyond," in "Scale invariance and Beyond," Proceedings of the CNRS workshop on scale invariance, Eds. B. Dubrulle, F. Graner, and D. Sornette, Springer, Berlin, 1997.

[26] P. Levy, Theorie de l'Addition des Variables Aleatoires (Gauthier-Villars, Paris, 1937).

[27] J. Hull, Options, Futures, and Other Derivatives (Prentice Hall, New Jersey, 1997). 
[28] R. Cont, Quantitative Finance, 1, 223 (2001).

[29] R. N. Mantegna and H. E. Stanley, An Introduction to Econophysics (Cambridge University Press, Cambridge, 2000).

[30] F. Black and M. Scholes, J. Political Economy, 81, 637 (1973).

[31] S. Ghashghaie, W. Breymann, J. Peinke, P. Talkner, and Y. Dodge, Nature, 381, 767 (1996).

[32] L. Borland, Phys. Rev. Lett., 89, 098701 (2002).

[33] A. Arneodo, J. F. Muzy, and D. Sornette, European Physics Journal B, 2, 277 (1998).

[34] G. Bellocchi, M. M. Dacorogna, C. M. Hopman, U. A. Müller, and R. B. Olsen, J. Emp. Finance, 6, 479 (1999).

[35] J.-P. Bouchaud, G. Iori, and D. Sornette, Risk, 9, 61 (1996). 